\title{
Fontes, aciers et alliages divers pour moulage de pièces devant résister à l'abrasion, à la corrosion, ou satisfaire à certaines caractéristiques
}

\author{
Cast irons, steels and various alloys \\ intended to resist abrasion or corrosion, \\ or to satisfy certain specifications
}

\author{
PAR R. POIROT \\ INGENTEUR E.E.M.I. ET E.S.F, \\ INGENIEUR AU CENTRE D'INFOHMATION DU NIGKEL
}

\begin{abstract}
Rappel sommaive des cas pouvant se présenter dans le choix d'un matérian pour des pièces de pompes, de turbines, etc. - Limitation de l'exposé à deux cas lypiques: «Abrasion》 el * Corrosion».

L'abrasion peut avoir un caractère peu sévère, et dans ce cas la fonte à graphite sphéroüdal perlitique est indiquée; l'abrasion penl au conlraire être très sévère: suivant que les pièces seront ou non usinés. et subiront ou non des chocs en service, on envisage les fontes martensitiques, soit grises, soit blanches, du type NiHard. Les píces de profil compliqué sont du domaine de lacier nickel-ehrome trempant ì l'air. Les compositions, les propriétés, les conditions de réception et le tratement de ces métaux sont ici définis.

La corrosion relève des fontes austéniliques du type Ni-Resist, des aciers inoxydables dn type 18/8. des alliages de cuivre et de nickel d̀ haule tenear en nickel dont le type est le métal Monel, ou encore des bronzes d'aluminium an nickel. Là encore, l'auteur indique les meilleures compositions, les curactéristiques, ainsi que les conditions d'emploi et d'usinage des divers alliages.
\end{abstract}

\begin{abstract}
A résume of problems likely to arise when choosing materials for components of machines such as farbines and pumps. Considerations limited to two typical cases; "Abrasion" and "Corrosion".

Abrasion may be slight and in such cases perlitic cast iron containing spheroidal graphile is indicated. On the other hand, where abrasion is acute, grey or Ni-Hard type white martensific cast irons are to be used, accorling to whether or not the component is to be machined, and whether or not it is to be subjected to shocks when in use. Components of complicated shape are to be made of air-hardened nickelchrominm steel. The composition, properties, acceplance tests, and treatment of these metals are defined.
\end{abstract}

Comosion makes necessary Ni-Resist Iype austenitic cast irons, $18 / 8$ type stainless steels, cooper-nickel allogs with a high nickel content as typified by Monel metal, or eoen aluminium bronzes containing nickel. Here again the anthor indicates the chatacteristics, the best composition as well as ways of using and machining these alloys.

\section{INTRODUCTION}

Le choix d'un matérian pour des pièces de pompes, de turbines, de matériel hydraulique, n'est pas toujours aisé car elles doivent souvent répondre à des conditions de service variées, en mettant même de côté les influences de l'abrasion, de la corrosion, de l'érosion et de la cavitation. Telle pièce devant résister à l'abrasion devra aussi avoir une résistance au choc acceptable, ou un bon frottement ou pouvoir être usinée avec précision; de même telle autre pièce aura à résister à la fois à l'abrasion el à la corrosion, telle autre encore, tout en résistant à la corrosion, devra avoir un bon frottement, ou une bonne résistance à la chaleur, ou ètre facilement moulable, etc... Faire une classification de tous les cas devant lesquels on peut se trouver et en déduire le matériau à utiliser serail une énumération difficile et fastidieuse. C'est pourquoi nous avons préféré considérer séparément les deux cas typiques * Abrasion » et 


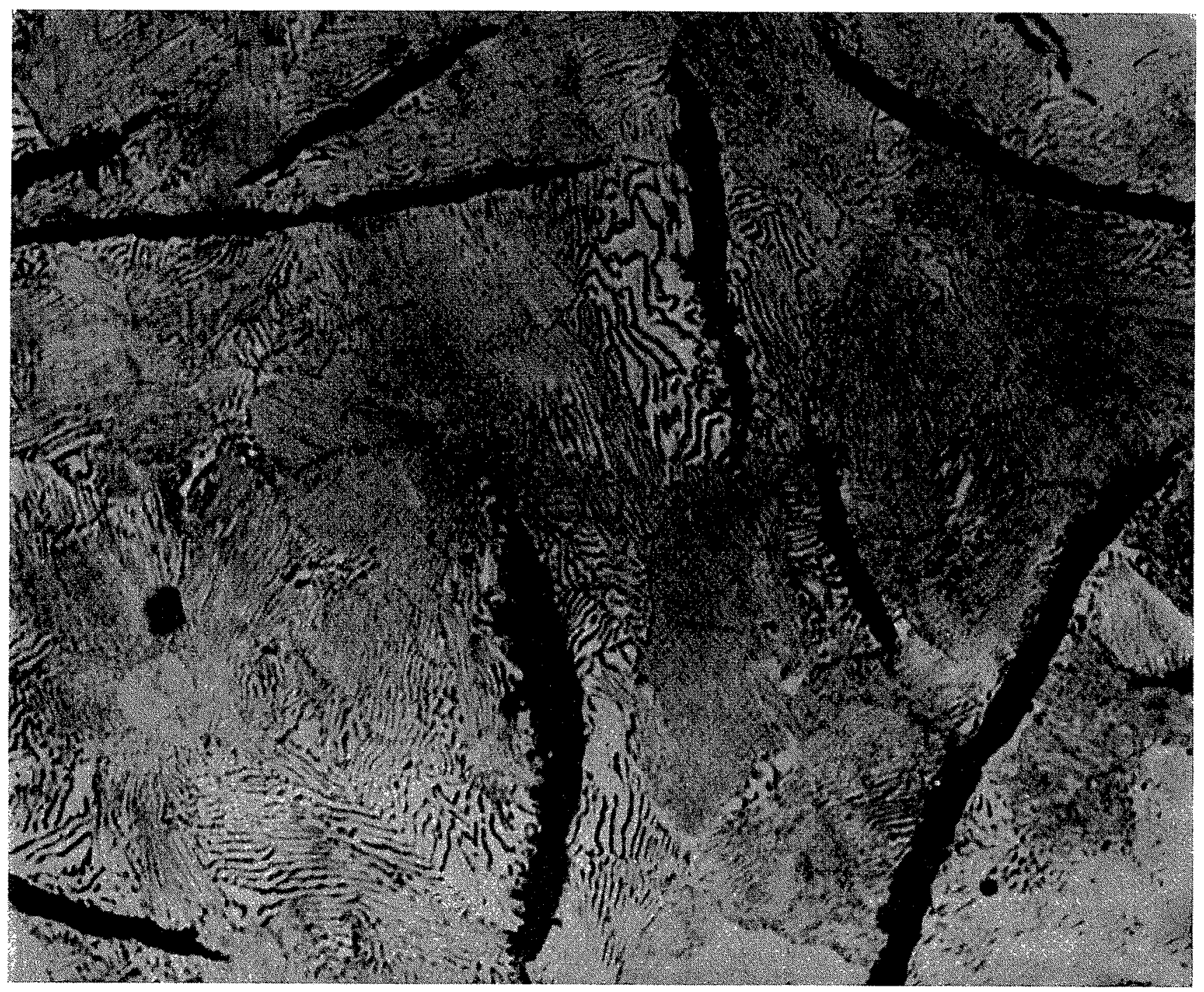

Frg. 1. - Fonte a graphite lamellaire. On voit nettement que les lamelles de graphite à extrémités aiguës entaillent la matrice et en diminuent aínsi les caractéristiques mécaniques.

« Corrosion » pour lesquels nous indiquerons quelques matériaux dont la qualité première est de résister principalement à l'un de ces phénomènes et dont nous essaierons de faire ressortir d'autres propriétés permettant de guider le constructeur pour le choix définitif de tel ou tel autre métal.
Les matériaux dont nous allons parler, les fontes alliées surtout, sont des métaux spéciaux qui demandent certains soins d'élaboration et de moulage, souvent de traitement thermique. Nous insistons donc dès à présent sur le fait que de tels métaux doivent être commandés à des fondeurs qui en ont l'expérience.

\section{I. - ABRASION}

Lorsque la question de résistance à l'abrasion est posée, la réponse est généralement l'emploi d'un matériau de dureté très élevée : la résistance à l'abrasion est généralement fonction de la dureté, mais il ne faut pas considérer cette carac- téristique comme seule indispensable. Ainsi, une même dureté peut être obtenue avec des matériaux de structures différentes; or, une structure peut avoir une meilleure résistance à l'abrasion qu'une autre qui, d'autre part, donne, par exem- 

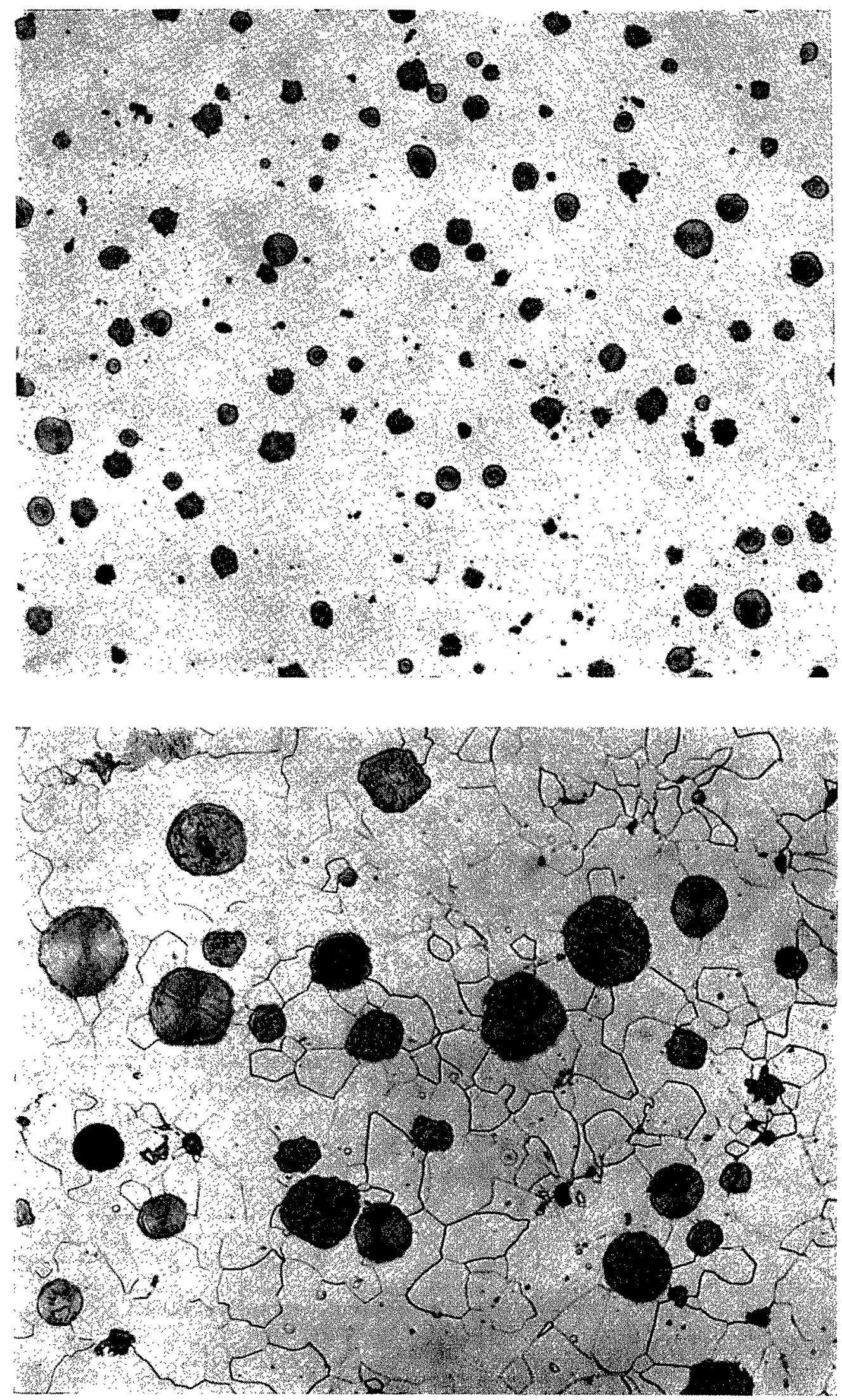

Fra. 2 et $2 \%$ - Fonte à graphite sphéroïdal. Le graphite cristallisé sous forme de sphères n'affecte plus les caractéristiques mécaniques de la matrice. 
ple, une dureté élevée au prix d'une grande fragilité.

D'autre part, le choix du matériau dépend bien entendu des conditions de service. Mais notre ćtude rapide sera surtout un examen des duretés et de la répercussion de celles-ci sur d'autres propriétés : usinabilité, résistance mécanique...; nous adopterons le plan suivant :

1. Abrasion ped sévère:

— Fonte à graphite sphéroïdal perlitique.

2. Abrasion sévìre :

a) Fontes grises d structure martensitique (les pièces doivent être usinées et ne subissent pas de chocs importants en service);

b) Fontes blanches martensitiques (type $\mathrm{Ni}$ Hard) (les pièces n'ont pas à être usinées et ne nécessitent pas une bonne résistance aux chocs).

c) Acier nickel-chrome trempant à lair (les pièces, d'un profil compliqué, doivent avoir une dureté élevée à cœur et une résistance mécanique élevée).

\section{1) Abrasion peu sévère}

Dans le cas d'abrasion peu sévère, il n'est peutêtre pas utile d'employer des matériaux très durs. Jusqu'ici on ne pouvait pas non plus songer à utiliser une fonte grise non alliée à graphite lamellaire, du fait de ses caractéristiques mécaniques faibles, sa fragilité et sa dureté peu élevée. Ces points faibles sont dus à la forme même du graphite, comme le montre la figure 1 .

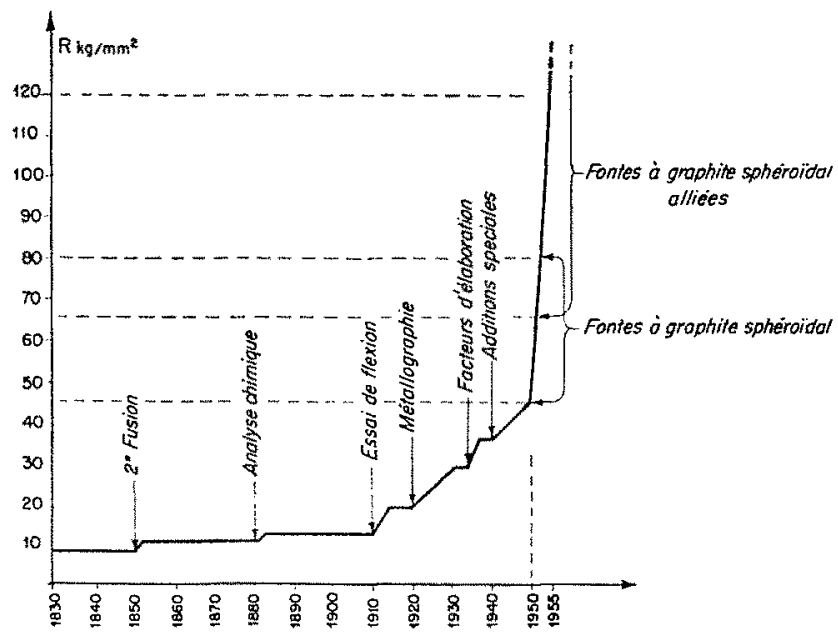

Fig. 3. - Evolution de la résistance à la traction des fontes notamment depuis ces trente dernières années. Cette courbe montre les fortes améliorations obtenues avec les fontes spéciales et les résistances remarquables obtenues avec les fontes à graphite sphéroïdal.
Un traitement spécial de la fonte, découvert il y a six ans environ, a permis de faire cristalliser le graphite sous forme de sphères $\left(^{*}\right)$ (fig. 2 et $2^{\prime}$ ). Cette fonte, dont le graphite n'entaille plus la matrice, a des caractéristiques mécaniques élevées (fig. 3) tout en conservant la facilité de moulage de la fonte grise ordinaire.

Elle peut avoir une structure soit perlitique obtenue à l'état brut de coulée (résistance à la traction élevée, allongements encore faibles), soit ferritique après recuit (résistance à la traction plus faible, mais allongements élevés), soit enfin ferrito-perlitique après recuit de ferritisation incomplet (caractéristiques mécaniques intermédiaires).

C'est avec une structure perlitique que la fonte à graphite sphéroïdal a les duretés les plus élevées; c'est done de ce type dont nous parlerons surtout : des propriétés mécaniques, de la tenuo à la pression, du frottement, du moulage, de l'usinabilité.

\section{Propriétés mécaniques :}

Les caractéristiques mécaniques à l'essai de traction de la fonte à graphite sphéroïdal perlitique peuvent varier dans ces limites:

$$
\begin{gathered}
\mathrm{R}=58-80 \mathrm{~kg} / \mathrm{mm}^{2}, \quad \mathrm{E}=42-60 \mathrm{~kg} / \mathrm{mm}^{2}, \\
\mathrm{~A}=0,5-5 \% ; \quad \mathrm{H}_{\mathrm{B}}=250-300 .
\end{gathered}
$$

Les caractéristiques minimum sont celles garanties par une spécification des fondeurs licenciés pour la production de ces fontes.

Les caractéristiques maximum sont celles que l'on peut atteindre après traitement de normalisation, c'est-à-dire chauffage à $900^{\circ} \mathrm{C}$ suivi d'un refroidissement à l'air calme.

La dureté de cette fonte peut s'échelonner, suivant la composition chimique, entre 250 et 300 Brinell. Dans le cas de pièces devant résister $\dot{a}$ une abrasion peu sévère, il sera préférable de choisir les nuances à plus fortes duretés, c'està-dire 280-300 Brinell.

D'autre part, la résistance au choc de la fonte à graphite sphéroïdal est supérieure à celle des meilleures fontes grises à graphite lamellaire. Pour une même structure perlitique, la résilience sur éprouvettes de $10 \times 10 \times 55 \mathrm{~mm}$ non entaillées et cassées sur appuis distants de $40 \mathrm{~mm}$, est de $1 \mathrm{kgm} / \mathrm{cm}^{2}$ environ pour une fonte à graphite sphéroïdal, c'est-à-dire bien supérieure au travail de rupture obtenu avec une fonte à graphite lamellaire.

(*) Ces nouvelles fontes ì graphite sphéroidal lont l'objet de breyets qui pour l'Europe, sont la propriété de la Mond Nickel Co, de Londres. 


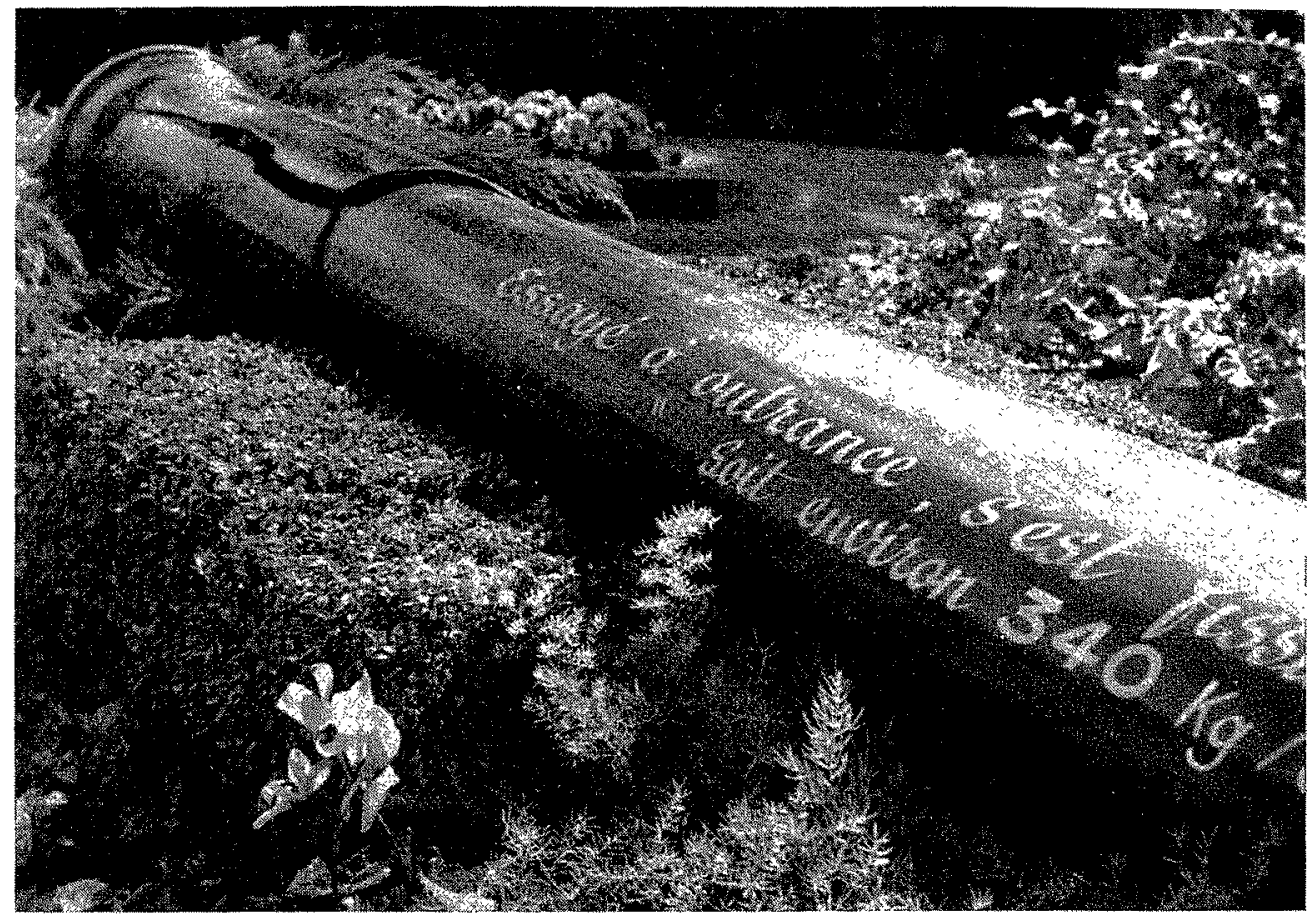

Fia. 4. - Tuyau centrifugé de $250 \mathrm{~mm}$ de diamètre intérieur après essai de pression à outrance. Il s'est fissuré pour une pression de $340 \mathrm{~kg} / \mathrm{cm}^{2}$.

\section{Trenue a la pression:}

EAgan et James $\left(^{*}\right.$ ) ont publié les résultats d'essais effectués sur des récipients à pression en fonte grise ordinaire, fonte alliée, acier moulé et fonte à graphite sphéroïdal; ces résultats indiquent que les pressions d'éclatement sont sensiblement proportionnelles à la charge de rupture du métal, ce qui situe bien la nouvelle fonte. Au cours d'essais de pression on a remarqué qu'elle n'éclatait pas en fragments, mais qu'elle se fissurait après gonflement (fig. 4); citons, par exemple, des essais de pression effectués sur des tuyaux centrifugés de $11 \mathrm{~mm}$ d'épaisseur qui, en fonte à graphite sphéroïdal; n'ont cédé que sous une pression de $340 \mathrm{~kg} / \mathrm{cm}^{2}$, alors que ceux en fonte ordinaire se rompent sous 150 à $200 \mathrm{~kg} / \mathrm{cm}^{2}$.

\section{$3^{\circ}$ Froterement :}

Le passage de la forme lamellaire à la forme sphéroïdale du graphite ne semble guère modifier cette qualité. A même quantité de graphite et à dureté semblable, la fonte à graphite sphéroïdal a sensiblement le même frottement que la fonte à graphite lamellaire.

(*) EAgan et James : A practical Enaluation of Ductile Cast Iron. Iron Age, 1949, CLXIV, déc. 8, 75-79, déc. 15, 77, 82 .
La matrice de la fonte a aussi une influence sur cette propriété et, pour un bon frottement, il

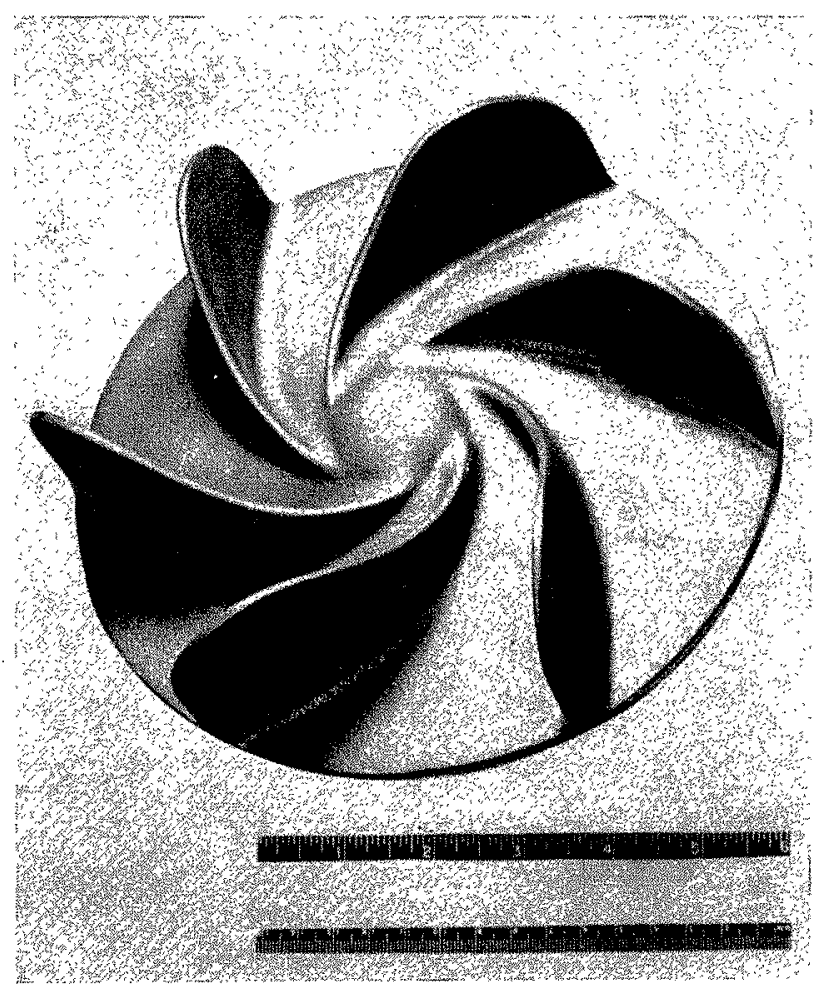

Fo. 5. - Impulseur de ventilateur en fonte a graphite sphéroidal. Les ailettes de $3 \mathrm{~mm}$ d'eptisseur sont lrès bien venues de fonderie. 
est recommandé d'utiliser une fonte à structure perlitique.

\section{$4^{\circ}$ Moulage :}

Tous les moulages de petites (fig. 5) ou fortes (fig. 6) dimensions normalement coulés en fonte, acier ou non ferreux, peuvent être exécutés en fonte à graphite sphéroïdal. Néanmoins, il est important de signaler que son aptitude à la retassure est nettement plus marquée que celle de la fonte ordinaire. Aussi le constructeur aurat-il intérêt, chaque fois que cela sera possible, à éviter dans le profil des pièces tous les changements brusques de section et la présence de masses importantes.

\section{$5^{\circ}$ USINABILITE :}

Malgré sa dureté plus élevée, la fonte à graphite sphéroïdal s'usine anssi bien que la fonte ordinaire. Par exemple la durée de l'outil est à peu près la même avec une fonte à graphite sphéroïdal de 220 Brinell et une fonte grise ordinaire de 145 Brinell. Néanmoins, la consom- mation d'énergie est supérieure pour la fonte à graphite sphéroïdal.

Le poli de surface est, d'autre part, bien supérieur à celui de la fonte ordinaire et semblable plutôt à celui de l'acier.

\section{2) Abrasion sévère}

a) Les pièces doivent être usinées et ne subissent pas de chocs importants en service :

FONTES GRISES MARTENSITIQUES :

Le nickel est un graphitisant, mais en outre il a une action sur la matrice de la fonte. Avec des additions croissantes de nickel, la dureté commence par croittre, passe par un maximum, puis décrô̂t, ce qui correspond à une modification de structure, c'est-à-dire au passage de la perlite à la sorbite, puis à la martensite, puis enfin à l'austénite. Les duretés les plus élevées correspondent à des teneurs en nickel de 4 à $10 \%$, c'est-à-dire à une structure martensitique.

Il est, d'autre part, nécessaire de régler la teneur en nickel en fonction de l'épaisseur du moulage, ainsi, évidemment, que celles en silicium et chrome. Le tableau I donne les com-

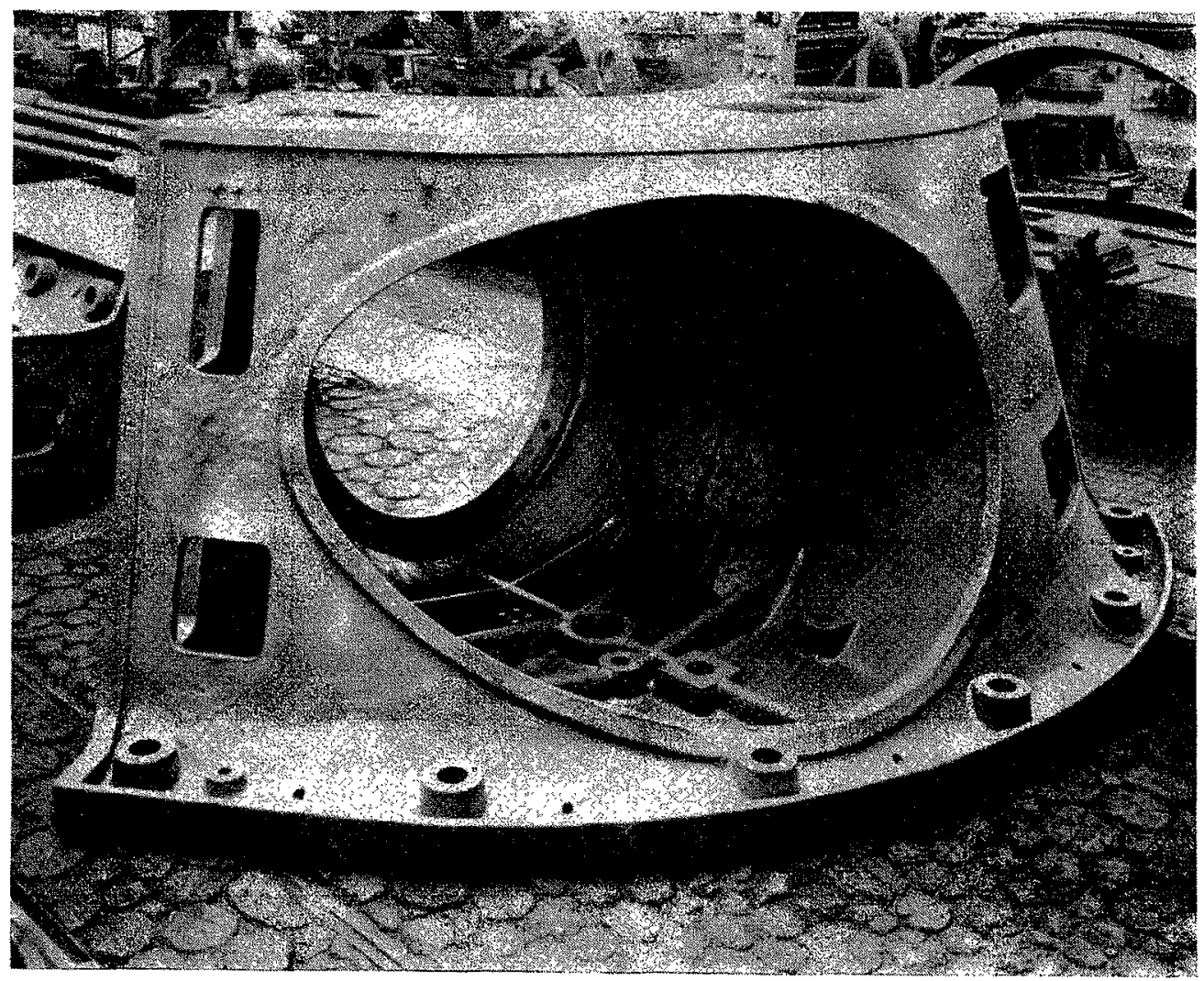

Fıg. 6. - Superstructure de turbine Pelton. Ce moulage de 11 tonnes a été coulé en fonte à graphite sphéroïdal. 


\section{TABLEAU I}

\begin{tabular}{|c|c|c|c|c|c|}
\hline \multirow{2}{*}{$\begin{array}{l}\text { Epaisseur } \\
\text { des pièces }\end{array}$} & \multicolumn{5}{|c|}{ COMPOSITION CHMLUUE } \\
\hline & $\mathrm{C}_{\mathrm{T}}$ & $\mathrm{Si}$ & Mn & $\mathrm{Ni}$ & $\mathrm{Cr}$ \\
\hline 5 à $10 \mathrm{~mm}$ & $3-3,3$ & $2,5-1,8$ & $0,8-1$ & $3,5-4$ & $0,3-0,8$ \\
\hline 10 à $15 \mathrm{~mm}$ & - & $\because, 2-1,6$ & - & $3,8-4,5$ & $0,5-1$ \\
\hline 15 à $20 \mathrm{~mm}$ & - & $1,8-1,4$ & -- & $4 \quad-4,5$ & $0,7-1$ \\
\hline 20 à $50 \mathrm{~mm}$ & -- & $1,5-1,2$ & … & $4,2-5$ & $0,8-1,2$ \\
\hline
\end{tabular}

\section{TABLEAU II}

\begin{tabular}{|c|c|c|c|c|c|c|c|}
\hline $\mathrm{C}_{\mathrm{T}}$ & Si & $\mathrm{Mn}$ & $\mathrm{Ni}$ & $\mathrm{Cr}^{\circ}$ & 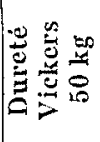 & 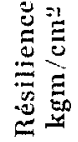 & $\begin{array}{l}\text { Micro- } \\
\text { structure }\end{array}$ \\
\hline 3,57 & 0,56 & 0,43 & 0 & 0 & 540 & 0,16 & $\begin{array}{l}\text { carbure } \\
\text { +perlite }\end{array}$ \\
\hline 3,57 & 0,56 & 0,43 & 0 & 1,95 & 576 & 0,21 & $\begin{array}{l}\text { carbure } \\
+ \text { perlite }\end{array}$ \\
\hline 2,90 & 0,48 & 0,36 & 4,35 & 1,70 & 593 & 0,43 & $\begin{array}{l}\text { carbure } \\
\text { + martensite } \\
\text { +austénite }\end{array}$ \\
\hline
\end{tabular}

TABLEAU III

\begin{tabular}{|c|c|c|c|c|c|c|c|}
\hline $\mathrm{C}^{\prime}$ & Si & $\mathrm{Mn}$ & $\mathrm{Ni}$ & $\mathrm{Cr}$ & 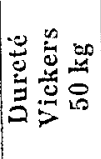 & 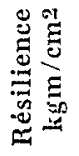 & $\begin{array}{l}\text { Micro- } \\
\text { structure }\end{array}$ \\
\hline 3,57 & 0,56 & 0,43 & 0 & 0 & 540 & 0,16 & $\begin{array}{l}\text { carbure } \\
+ \text { perlite }\end{array}$ \\
\hline 3,57 & 0,50 & 0,43 & 0 & $1,9 j$ & 576 & 0,21 & $\begin{array}{l}\text { Larbure } \\
+ \text { perlite }\end{array}$ \\
\hline 3,57 & 0,56 & 0,56 & 3,80 & 1,95 & 6155 & 0,28 & $\begin{array}{l}\text { carburc } \\
\text { + martensite } \\
+ \text { un peu } \\
\text { d'austénite }\end{array}$ \\
\hline
\end{tabular}

positions préconisées pour des moulages d'épaisseur moyenne allant de 5 à $50 \mathrm{~mm}$.

Ces fontes, de composition correctement choisie, donnent des moulages inusinables à l'état brut de coulée, mais un revenu à $600-625^{\circ} \mathrm{C}$ les adoucit et permet ainsi leur usinage. - Après usinage, les moulages peuvent être à nouveau durcis par chauffage à $850^{\circ} \mathrm{C}$ suivi d'un refroidissement à l'air calme. Il est recommandé de faire suivre ce traitement d'un revenu dे 250$300^{\circ} \mathrm{C}$ pour détruire les tensions internes qui peuvent se produire au cours de la trempe martensitique. Après ces traitements, les moulages ont des duretés de l'ordre de 350-400 Brinell.

Comme pour les fontes non alliées, le graphite peut être, aussi dans ce cas, lamellaire ou sphéroïdal. Le graphite sphéroïdal permet d'obtenir des caractéristiques mécaniques élevées, puisque l'on a atteint jusqu'à $120 \mathrm{~kg} / \mathrm{mm}^{2}$, et des duretés de l'ordre de 450-500 Brinell.

b) Les pièces n'ont pas à être usinées et ne nécessitent pas une bonne résilience au choc:

FONTES BLANCHES MARTENSITIQUES DU TYPE NI-HARD :

- Les fontes blanches martensitiques permettent d'obtenir les mêmes duretés qu'avec les fontes blanches non alliées à structure perlitique, mais avec une quantite moindre de carbure et donc avec moins de fragilifé (Tableau II).

- A quantité équivalente de carbure, les fontes blanches martensitiques ont des duretés plus élevées que les fontes blanches perlitiques ('Tableau III).

Donc pour une bonne résistance à l'abrasion, selon que la pièce en service risque ou ne risque pas de subir des chocs, on peut employer une fonte blanche martensitique à bas carbone, c'est-à-dire avec moins de carbure et par suite moins fragile, ou une fonte blanche martensitique plus carburée, à haut carbone, c'est-à-dire à dureté élevée.

\section{Compositions :}

Comme pour les fontes grises martensitiques, la teneur en nickel doit être réglée en fonction de l'épaisseur du moulage. Elle doit être moindre dans les moulages minces pour ne pas $y$ avoir d'austénite résiduelle, et plus élevée dans les moulages épais pour ne pas y avoir de perlite.

Il faut aussi régler la teneur en nickel en fonction de la teneur en carbone.

De même, les teneurs en silicium et chrome seront déterminées par rapport à l'épaisseur du moulage. Le chrome a pour rôle principal de contre-balancer l'action graphitisante du nickel.

Tenant compte de ces considérations, nous donnons pour des pièces coulées en sable, dans le tableau IV, les compositions de fonte Ni-Hard préconisées en fonction des épaisseurs des moulages et de la teneur en carbone. 
TABLEAU IV

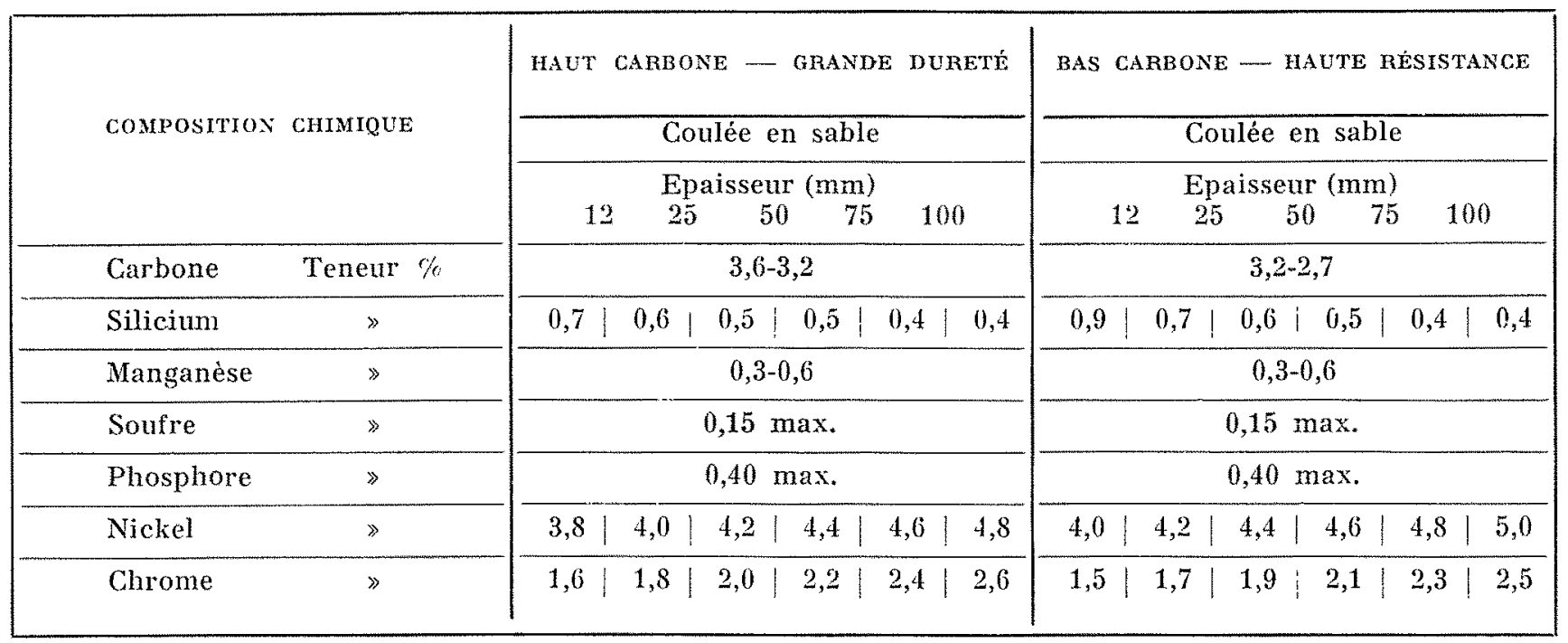

CONDITIONS DE RÉCEPTION :

$\mathrm{Si}$ ces compositions ont bien été respectées par le fondeur en fonction de l'épaisseur des monlages, les pièces coulées en sable doivent avoir des duretés de 550-650 Brinell pour celles à haut carbone et 525-625 Brinell pour celles à bas carbone.

Ces duretés doivent être mesurées avec une bille en carbure de tungstène soit de $10 \mathrm{~mm}$ de diamètre sous une pression de $3.000 \mathrm{~kg}$, soit de $5 \mathrm{~mm}$ de diamètre sous une pression de $750 \mathrm{~kg}$.

D'autre part, les pièces coulées en fonte blanche martensitique du type Ni-Hard, comme celles coulées en fonte blanche ordinaire d'ailleurs, sont soumises à des tensions internes qui les rendent fragiles. Ces tensions cependant peuvent être facilement éliminées par un simple traitement de détente. Les pièces doivent donc être livrées après avoir subi un revenu à 200$230^{\circ} \mathrm{C}$ pendant trois heures, plus une heure par $25 \mathrm{~mm}$ d'épaisseur supplémentaire. Ce revenu n'altère pas la dureté des pièces.

c) Les pièces, d'un profil compliqué, doivent avoir une dureté élevée à cour et une résistance mécanique
élevée :

ACIER NICKEL-CHROME TREMPANT A L'AIR :

Nous citerons pour mémoire quelques nuances d'aciers bien connues comme susceptibles de donner des durelés élevées dans les moulages:

$1^{\circ}$ Acier à $13 \%$ de chrome : sa dureté peut être augmentée par trempe à l'huile, mais pour des moulages compliqués on doit craindre les tapures; $2^{\circ}$ Acier au chrome-molybdène contenant du nickel pour abaisser la vitesse critique de trempe;

$3^{\circ}$ Acier's autotrempants au nickel-chrome qui sont les plus importants et permettent d'obtenir dans les pièces des duretés à cœur élevées sans risque de tapures.

La composition de ces aciers peut varier dans les limites suivantes :

$$
\begin{array}{r}
\mathrm{C}_{\mathrm{T}}-0,25-0,60 \\
\mathrm{Mn}-0,60-0,90 \\
\mathrm{Ni}-2,80-4,50 \\
\mathrm{Cr}-0,80-1,50
\end{array}
$$

Ces aciers contiennent aussi la plupart du temps du molybdène qui a tendance à augmenter sensiblement l'autotrempe et diminue efficacement les phénomènes de fragilité après revenu ou après refroidissement lent. La teneur en cet élément est généralement de 0,2 à $0,5 \%$.

Le traitement thermique des pièces consiste, après recuit d'homogénéisation, en une trempe à l'air après chauffage à $850^{\circ} \mathrm{C}$ suivi d'un revenu. Selon la température du revenu, les caractéristiques mécaniques peuvent varier dans les limites suivantes:

$$
\begin{aligned}
& \mathrm{R}=90-170 \mathrm{~kg} / \mathrm{mm}^{2} \\
& \mathrm{E}=75-140 \mathrm{~kg} / \mathrm{mm}^{2} \\
& \mathrm{~A}=6-16 \% \\
& \mathrm{KE}_{2}=5-14 \mathrm{kgm} / \mathrm{cm}^{2} \\
& \mathrm{H}_{3}=275-500
\end{aligned}
$$




\section{II. - CORROSION}

Le problème de la rćsistance à la corrosion est aussi très complexe et a fait l'objet de nombreuses et longues études sur une grande diversité de matériaux. Nous nous bornerons, ici, a citer ceux qui sont les plus couramment employés et que nous avons étudiés plus particulierement :

- les fontes austénitiques du type Ni-Résist,

- les aciers inoxydables du type $18 / 8$,

- les cupro-nickel, et, en particulier, le métal Monel.

\section{Fontes austénitiques du type Ni-Resist}

Il existe plusieurs types de fontes austénitiques (Ni-Résist) (fig. 7) dont nous donnerons, en plus de leurs compositions, quelques indications sur le moulage, l'usinabilité, les propriétés mécaniques, la résistance à la chaleur.

\section{$1^{\circ}$ Compositions :}

Les compositions sont indiquées dans le tableau $\mathrm{V}$ ci-après.

Chacune de ces compositions confère à la fonte une particularité d'emploi. Les types I et II sont les plus généralement utilisés.

Type I. Il est recommandé pour la manutention d'acides minéraux dilués.

Type II. II résiste à un grand nombre de milieux corrosifs et du fait de sa basse teneur en cuivre, peut être employé dans les cas où cet élément est indésirable.

Typs I a et II $b$. A basse teneur en carbone, ils ont une honne résistance mécanique et peu- vent être utilisés pour des pièces devant résister à la pression.

Type II b. Ce type à haute teneur en chrome peut être employé surtout pour résister à des températures allant jusqu’à $810-820^{\circ} \mathrm{C}$. En outre, la dureté plus élevée (170-250 Brinell) de ce type lui confère une meilleure résistance à l'abrasion.

Type HI. Il est recommandé dans le cas où les moulages sont soumis à des chauflages et refroidissements répétés entre la température ambiante et $225^{\circ}$, car il a un faible coefficient de dilatation.

Type IV. Il est supérieur aux autres types au point de vue résistance à la chaleur et à la corrosion.

Type Y. Appelé « MINVAR ». Il est surtout utilisé pour son très faible coefficient de dilatation.

\section{$2^{\circ}$ Moulage :}

L'aptitude à la retassure de ces fonles est très marquée et semblable à celle de l'acier. Pour le tracé des pièces on prendra donc Jes mêmes précautions que pour celui des moulages d'acier; tous les changements brusques de sections sont à éviter. Lorsqu'il s'agit de couler avec cette fonte une pièce habituellement exéculée en un autre métal a caractéristique de solidification différente, on devra laisser au fondeur toute latitude pour modifier le modèle et assurer ine alimentation correcte du moulage.

En outre, le retrait de cetle fonte est clevé et de l'ordre de $16 \%$.

TABLEAU $V$

\begin{tabular}{|c|c|c|c|c|c|c|c|c|c|}
\hline & & Type I & Type I $a$ & Type II & Type II $a$ & Type II $b$ & Type III & Type IV & Type V \\
\hline Carbone & $\%$ & $3 \max$. & $2,80 \max$ & $3 \max$ & $2,80 \max$ & $3 \max$ & $2,75 \max$ & $2,60 \max$ & $2,40 \max$ \\
\hline Silicium & $\%$ & $-2,5$ & $1,5-2,75$ & $\begin{array}{ll}1 & -2,5\end{array}$ & $1,5-2,75$ & $1-2,5$ & $1-2$ & $5-6$ & $1-2$ \\
\hline Mangarièse & $\%$ & $1-1,5$ & $1-1,5$ & $0,8-1,5$ & $0,8-1,5$ & $0,8-1,5$ & $0,4-0,8$ & $0, \mathbf{4}-0,8$ & $0,4-0,8$ \\
\hline Nickel & $\%$ & $13,5-17,5$ & $13,5-17,5$ & $\begin{array}{ll}18 & -22\end{array}$ & $\begin{array}{ll}18 & -22\end{array}$ & $\begin{array}{ll}18 & -22\end{array}$ & $\begin{array}{ll}28 & -32\end{array}$ & $29-32$ & $34-36$ \\
\hline Cuivre & $\%$ & $5,5-7,5$ & $5,5-7,5$ & $0,5 \max$ & $0,5 \max$ & $0,5 \max$ & $0,5 \max$ & $0,5 \max$ & $0,5 \max$ \\
\hline Chrome & $\%$ & $1,75-2,5$ & $1,75-2,5$ & $1,75-2,5$ & $1,75-2,5$ & $3-6$ & $2,5-3,5$ & $4,5-5,5$ & $0,10 \max$ \\
\hline
\end{tabular}






FIa. 7. - Corps de vannes et couvercles en fonte austẻnitique du type Ni-Résist.

\section{$3^{\circ}$ USINABILITÉ :}

L'usinage à bon marché de la fonte Ni-Résist la fait souvent préférer à un autre matériau résistant bien à la corrosion mais d'un usinage plus coûteux.

Pour une dureté normale de 130-160 Brinell, la Nî-Résist s'usine très bien. Il est recommandé d'utiliser dans ce cas des outils moulés en alliage non ferreux tels que stellite $98-\mathrm{M}_{2}$ qui permettent des vitesses de coupe de 10 à $15 \%$ supérieures à celles adoptées avec l'acier rapide.

Pour des duretés supéricures à 175 Brinell, il faut utiliser des outils aux carbures en réduisant les vitesses sans diminuer beaucoup les avances.

Au-delà de 240 Brinell, il faut utiliser le meulage.

\section{PRopriétés MÉcanigues :}

La charge de rupture à la traction, pour la plupart des types de cette fonte, varie de 17 à $25 \mathrm{~kg} / \mathrm{mm}^{2}$. Pour les types I $a$ et II $a$ à bas carbone, elle peut atteindre $35 \mathrm{~kg} / \mathrm{mm}^{2}$.

La dureté est de l'ordre de 130-180 Rrinell. Elle peut atteindre 250 Brinell pour des teneurs en chrome élevées, mais dans ce cas l'usinage devient difficile.
Comme pour les fontes ordinaires, les fontes grises austénitiques peuvent avoir leur graphite sous forme de sphères, ce qui leur confère des caractéristiques mécaniques intéressantes. On a pu obtenir ainsi, par exemple, de $38 \div 43 \mathrm{~kg} / \mathrm{mm}^{2}$ de charge de rupture à la traction avec 10 a $20 \%$ d'allongement. La limite élastique, inexistante chez les fontes grises austénitiques à graphite lamellaire, peut atteindre pour ces fontes à graphite sphéroildal $24-25 \mathrm{~kg} / \mathrm{mm}^{2}$. En outre, quelle que soit la forme du graphite, la résistance d̀ la corrosion reste sensiblement la mêrne.

\section{$5^{\circ}$ RÉsistance A LA CHALEUR :}

Les fontes austénitiques ont une résistance à l'oxydation qui peut aller jusqu'à Jix fois celle de la fonte ordinaire et une résistance au gonflement pouvant être supérieure de douze fois, pour des températures de 700 à $815^{\circ}$.

Cependant, les fontes Ni-Résist ne sont pas recommandées pour des températures supérieures à $800^{\circ}$, et, d'autre part, lorsque les produits véhiculés contiennent quantité de soufre. la limite d'utilisation se tient vers $550^{\circ}$. Par contre, le type IV, résistant au ternissement, a une meilleure tenue à des températures plus élevées et même en présence de soufre. 


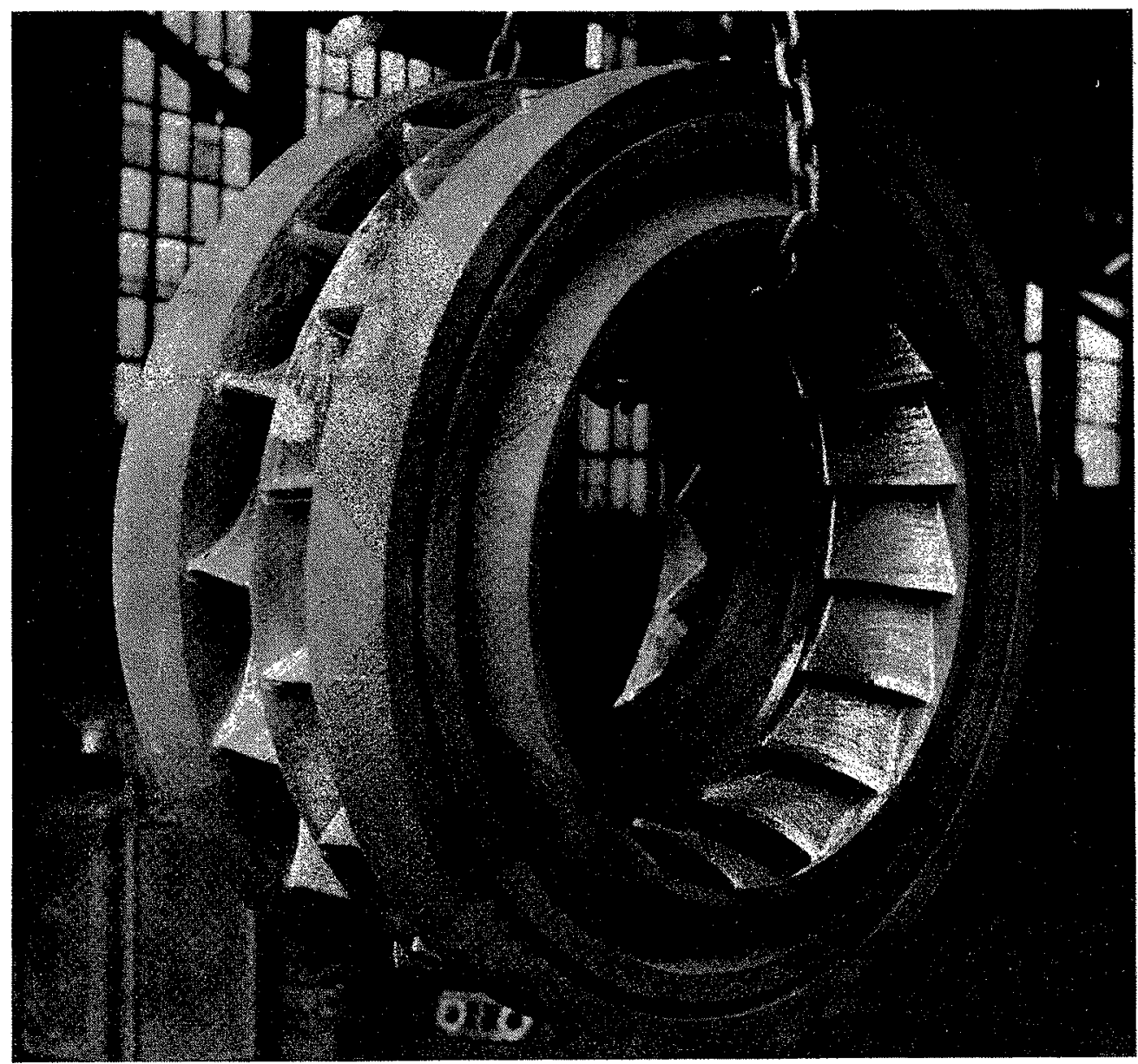

Fra. 8. - Turbine Francis en acier inoxydable $\mathrm{Ni}-\mathrm{Cr}^{*}$ austéno-ferritique: Poids brut $3200 \mathrm{~kg}$ (Jacob Holtzer).

\section{Aciers inoxydables}

Movlage ET Compositrons :

Les aciers inoxydables austénitiques (fig. 8) sont plus difficiles à mouler que les aciers ordinaires, du fait d'une rapide pellicule qui se forme à la surface du bain, de leur mauvaise coulabilité, et de leur retrait important qui peut atteindre $25 \%$. La coulabilité peut être améliorée par une augmentation des teneurs en carbone et en silicium. Une teneur en carbone plus élevée présente cependant l'inconvénient de nécessiter un traitement de mise en solution des carbures qui peut être délicat pour certaines pièces compliquées.

Devant les difficultés de moulage de ces aciers, il est impératif que le constructeur s'efrorce pour le fondeur à uniformiser les épaisseurs des pièces.

Les deux compositions types pour le 18/8 moulé sont:

$$
\begin{array}{lllllllll}
\% & \mathrm{C} \leqslant 0,7 . & \mathrm{Cr} \geqslant 18 . & \mathrm{Ni} \geqslant 8 . & \mathrm{Mn} \leqslant 1 . & \mathrm{Si} \leqslant 2 . & \mathrm{S} \text { et } \mathrm{P} \leqslant 0,05 . & \mathrm{Mo} 2 \text { à } 4 \\
\% & \mathrm{C} \leqslant 0,20 . & \mathrm{Cr} \geqslant 18 . & \mathrm{Ni} \geqslant 8 . & \mathrm{Mn} \leqslant 1 . & \mathrm{Si} \leqslant 1 . & \mathrm{S} \text { et } \mathrm{P} \leqslant 0,05 . & \mathrm{Mo} 2 \text { a } 4
\end{array}
$$

Ơn peut obtenir : $R \geqslant 50 \mathrm{~kg} / \mathrm{mm}^{2} \quad \mathrm{E} \geqslant 23 \mathrm{~kg} / \mathrm{mm}^{2} \quad A \geqslant 40 \%$, sur $50 \mathrm{~mm} \quad \mathrm{H}_{\mathrm{B}} \leqslant 200$

Il est possible d'améliorer ces caractéristiques mécaniques sans changer beaucoup la résistance à la corrosion en utilisant soit des aciers $18 / 8$ contenant un ou plusieurs éléments : silicium, cuivre, titane, bore, aluminium... et employés après un traitement thermique dit de durcissement structural, soit des aciers à plus forte teneur en chrome (sans changer la teneur en nickel ou en l'abaissant) à structure austéno-ferritique et qui peuvent aussi subir le traitement de durcissement structural. 


\section{Alliages à haute teneur en nickel}

\section{Le métal Monel :}

Ce métal est un alliage nickel-cuivre comprenant environ deux tiers de nickel et un tiers de cuivre. Il résiste bien à un bon nombre d'agents corrosifs tels que le sel marin, les alcalis caustiques, l'acide sulfurique, le sulfate d'ammonium... et il conserve, aux températures élevées, une bonne partie de sa résistance.

Sa composition est comprise dans les limites suivantes :

$\mathrm{Ni}=67 \%, \quad \mathrm{Cu}=28 \%, \quad \mathrm{Fe}=$ moins de $3,5 \%$, $\mathrm{Si}=0,75-2 \%, \mathrm{C}=0,15-0,25 \%$,

$\mathrm{Mn}=0,50 \%$ environ, $\mathrm{S}=$ le moins possible.

Les caractéristiques mécaniques de ce métal sont comprises dans les limites suivantes :

$$
\begin{gathered}
\mathrm{R}=36-52 \mathrm{~kg} / \mathrm{mm}^{2}, \quad \mathrm{E}=17-24 \mathrm{~kg} / \mathrm{mm}^{2}, \\
\mathrm{~A}=16-40 \%, \quad \mathrm{H}_{\mathrm{B}}=100-140 .
\end{gathered}
$$

LES CUPRO-NICKELS A L'ÉTAIN ET AU SILICIUM :

Ces alliages peuvent être intéressants pour des pièces devant avoir une grande dureté à froid ou à chaud, et un bon frottement. Leur composition varie dans les limites suivantes :

$\mathrm{Ni}=50$ à $65 \%, \mathrm{Sn}=2$ à $12 \%, \mathrm{Si}=1$ à $3 \%$, Impuretés $=1$ à $3,5 \%(\mathrm{Fe}, \mathrm{Mn} . .),. \mathrm{Cu}=$ le solde.

Ils ont une dureté pouvant dépasser 350 Brinell et qui reste à peu près constante pour des températures allant jusqu'à $600^{\circ}$.

Ils sont formés d'une matrice relativement molle dans laquelle sont noyés des cristaux très durs semblables aux constituants des bronzes et d'autant plus nombreux que les teneurs en étain et silicium sont élevées. On est cependant limité pour les teneurs en ces éléments car ils ont tendance à favoriser les retassures dans les pièces.

\section{Hastelloys :}

Pendant longtemps le problème de résistance à la corrosion par des produits très actif́s acides chlorhydrique, sulfurique, phosphorique) est resté sans solution pratique. La mise au point d'alliages nickel-molybdène ou chrome-nickelmolybdène, appelés Hastelloys, a pu résoudre cette question. Il en existe de nombreuses compositions.

L'Hastelloy C, par exemple, de composition : $\mathrm{Cr}=14 \%, \mathrm{Ni}=58 \%, \mathrm{Mo}=17 \%, \mathrm{Fe}=6 \%$, a, à l'état moulé, les caractéristiques mécaniques suivantes :

$$
\begin{gathered}
\mathrm{R}=52-56 \mathrm{~kg} / \mathrm{mm}^{2}, \quad \mathrm{E}=31-33 \mathrm{~kg} / \mathrm{mm}^{2}, \\
\mathrm{~A}=10-15 \%, \quad \mathrm{H}_{\mathrm{B}}=175-215 .
\end{gathered}
$$

Il résiste bien dans les solutions chlorhydri- ques et sulfuriques contenant des sels oxydants et jusqu'à des températures de $70^{\circ}$ environ; de même, il a une bonne tenue en présence de chlore humide.

\section{LFS BRONZES D'ALUMINIUM AU NICKEL :}

Le nickel augmente la résistance mécanique et améliore les résistances à la corrosion, à l'usure et à la chaleur des bronzes d'aluminium pour des additions allant jusqu'à $5 \%$. Ces bronzes spéciaux ont, en outre, une bonne résistance ì la cavitation, à l'érosion et à la fatigue sous corrosion.

Le type de bronze d'aluminium au nickel le plus employé pour les moulages a la composilion suivante:

$$
\begin{gathered}
\mathrm{Cu}=78 \%, \quad \mathrm{Al}=10 \%, \quad \mathrm{Fe}=4 \%, \\
\mathrm{Mn}=3,5 \%, \quad \mathrm{Ni}=5,5 \% \max .
\end{gathered}
$$

Les caractéristiques mécaniques de cet alliage sont :

\begin{tabular}{|l|c|c|}
\hline & Brul de conlee & Traité \\
\hline R en $\mathrm{kg} / \mathrm{mm}^{2} \ldots \ldots \ldots$ & $60-65$ & $78-80$ \\
E en $\mathrm{kg} / \mathrm{mm}^{2} \ldots \ldots \ldots$ & $28-30$ & $43-45$ \\
A en $\% \ldots \ldots \ldots \ldots$ & 6 & 5 \\
H Brinell.......... & 190 & 200 \\
\hline
\end{tabular}

Par trempe et revenu, la résistance à la traction et la dureté de ce bronze d'aluminium au nickel peuvent ètre augmentées aux dépens de la ductilité, comme l'indiquent les chiffres cidessus. Généralement, cet alliage est utilisé à l'état brut de coulée, notamment pour des moulages importants ou compliqués. Pour des moulages plus simples où l'on désire augmenter la duretí, tels que siège et clapets de vannes, le traitement thermique peut être intéressant.

Les bronzes d'aluminium au nickel ont une très bonne résistance à la corrosion par l'eau douce ou l'eau de mèr. En outre, l'alliage dont nous avons donné la composition ci-dessus résiste bien à la cavitation ou à l'érosion. Il convient donc bien pour des impulseurs de pompes, corps de vannes ou pompes.

Les bronzes d'aluminium au nickel conservent en grande partie leur résistance mécanique à des températures pouvant atteindre $400^{\circ} \mathrm{C}$. Cette propriété, liée à leurs bonnes résistances à la corrosion et à l'oxydation, les rend propres à être utilisés en présence de vapeur's, dans les moteurs 
à combustion interne, et pour certaines applications de l'industrie chimique.

Enfin, du fait de leur dureté relativement élevée, les bronzes d'aluminium au nickel peuvent ètre employés pour des pièces devant résister à l'usure soit par frottement à sec ou avec un peu de lubrification, soit par abrasion. Signalons d'autre part que ces bronzes conservent leur dureté à des températures plus élevées que les bronzes ordinaires.

$$
* * *
$$

Nous venons de passer en revue quelques-uns des métaux susceptibles de résister à l'abrasion, à la corrosion, en essayant de faire ressortir, pour chacun d'eux, leurs propriétés essentielles permettant de guider le choix $d u$ constructeur. En dehors des nécessités de la mécanique, le choix du matériau, notamment pour l'hydraulique, est un cas précis, souvent un cas d'espèce où la nature des produits et certaines conditions de service sont une influence dominante et déterminante.

Dans ce texte, l'auteur a utilisé certaines données exposées au cours d'une conférence le 17 novembre 1954 , 10, avenue Hoche, devant les constructeurs de la Chambre Syndicale des Constructeurs de Pompes.

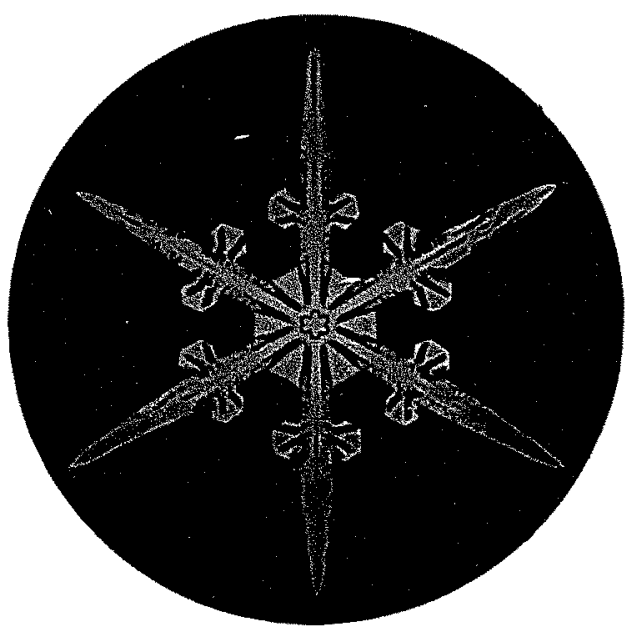

